den, nahm während der gesamten Beobachtungszeit deutlich zu. Entsprechend stieg auch der Anteil der Patienten mit einer Ulkusblutung, die niedrig dosiertes ASS einnahmen von 9,9\% in der ersten Periode auf $18,8 \%$ in der zweiten Periode.

\section{Kommentar}

Diese Daten bestätigen die alltäglichen Erfahrungen, dass der Anteil der Patienten mit einer oberen gastrointestinalen Blutung auch bei einer niedrig dosierten ASS-
Therapie in den letzten Jahren deutlich zugenommen hat. Entscheidend dafür dürfte die Tatsache sein, dass immer mehr Patienten mit einer kardiovaskulären oder zerebrovaskulären Erkrankung ASS als Thrombozytenaggregationshemmung erhalten. Daraus ergibt sich die Notwendigkeit, bei jedem Patienten nach entsprechender individueller Risikostratifizierung die Indikation für eine PPI-Prophylaxe zu diskutieren. Dazu gehören insbesondere betagte Patienten, Patienten mit einer gastrointestinalen Blutung in der Vorge- schichte, einer Komedikation mit NSAR, Kortison oder Marcumar. Zusätzlich vor Einleitung einer dauerhaften ASS-Therapie den Helicobacterstatus zu überprüfen und eventuell eine Eradikation durchzuführen, dürfte angesichts der großen Patientenzahlen kaum möglich sein.

P. Stiefelhagen

\footnotetext{
- M. Nakayama et al.

Low-dose aspirin is a prominent case of bleeding ulcers in patients who underwent emergency endoscopy. J. Gastroenetrol 44 912-918
}

\title{
Benzodiazepine können eine Ischialgie nicht lindern
}

\begin{abstract}
Rückenschmerzen und radikuläre Schmerzen sind außerordentlich häufig. In den meisten Leitlinien wird empfohlen, dass neben einer Behandlung mit Krankengymnastik, Physiotherapie und nicht steroidalen Antirheumatika (NSAR) auch Benzodiazepine als Muskelrelaxanzien eingesetzt werden sollten. Allerdings gibt es zum Einsatz der Benzodiazepine in dieser Indikation nahezu keine randomisierten prospektiven Studien.
\end{abstract}

- An der Neurologischen Universitätsklinik wurden 60 Patienten in eine randomisierte prospektive Studie eingeschlossen. Sie waren zwischen 18 und 75 Jahren alt, litten unter radikulären Schmerzen ohne neurologische Ausfälle und hatten einen im CT oder in der Kernspintomografie nachgewiesenen Bandscheibenvorfall. 30 Probanden erhielten Placebo und 30 zweimal $5 \mathrm{mg}$ Diazepam am Tag.

Der primäre Studienendpunkt war die Reduktion der Länge der Schmerzausstrahlung im betroffenen Bein. Sekundäre Endpunkte waren unter anderem die Beeinträchtigung, die Schmerzintensität, neurologische Ausfälle, die Gehstrecke und die Arbeitsfähigkeit.

Das mittlere Alter der Patienten betrug 42 Jahre, und die mittlere Schmerzdauer lag zwischen 14 und 21 Tagen. Bei den meisten Patienten lag ein Band-

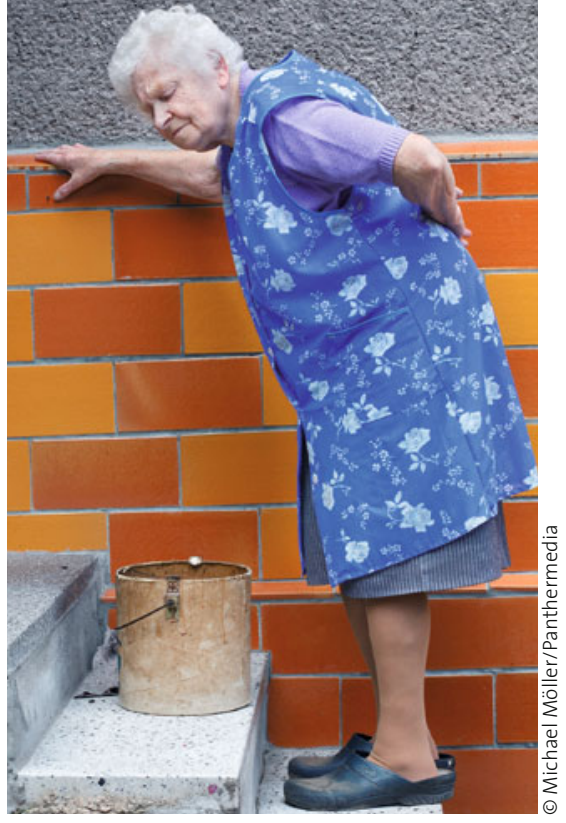

Kein Fall für Benzodiazepine.

scheibenvorfall L4/L5 oder L5/S1 vor. Die prozentuale Reduktion der Strecke der ausstrahlenden Schmerzen betrug im Mittel 60\% unter Placebo und 50\% unter Diazepam. Damit ergab sich ein statistisch signifikanter Vorteil zugunsten von Placebo. Für die übrigen Endpunkte zeigten sich keine Unterschiede zwischen Verum und Placebo. Die Aufenthaltsdauer im Krankenhaus war allerdings bei Patienten, die mit Placebo behandelt wurden, kürzer. Die Responderrate bezüglich der Schmerzreduktion von $50 \%$ oder mehr betrug bei diazepambehandelten Patienten $41 \%$ und bei placebobehandelten Patienten 79\% und war ebenfalls statistisch signifikant.

Schlussfolgerung: Benzodiazepine haben bei der Behandlung von radikulären Schmerzen bei Patienten mit lumbalen Bandscheibenvorfällen keinen Nutzen.

\section{Kommentar}

Es ist außerordentlich lobenswert und erfreulich, dass endlich eine Arbeitsgruppe die Aufgabe übernommen hat, den therapeutischen Wert von Benzodiazepinen bei Patienten mit lumbalen Bandscheibenvorfällen und radikulären Schmerzen in einer placebokontrollierten Studie zu untersuchen. Die Studienergebnisse sind ganz eindeutig: Für die meisten Parameter ergab sich kein Unterschied und für den primären Endpunkt ergab sich sogar eine Überlegenheit von Placebo versus Benzodiazepinen.

Die Empfehlung, dass Benzodiazepine bei Patienten mit diesen Beschwerden verordnet werden sollten, müsste daher aus den Leitlinien gestrichen werden.

H.-C. DienER *

- D. Brötz et al.

Is there a role for benzodiazepines in the management of lumbar disc prolapse with acute sciatica? Pain 149 (2010) 470-475 